PROCEEDINGS OF THE

AMERICAN MATHEMATICAL SOCIETY

Volume 139, Number 7, July 2011, Pages 2529-2541

S 0002-9939(2010)10793-1

Article electronically published on December 21, 2010

\title{
ON INJECTIVE VON NEUMANN ALGEBRAS
}

\author{
G. RACHER
}

(Communicated by Marius Junge)

\begin{abstract}
Partially answering a question of A.Ya. Helemskii, we show that a von Neumann algebra is injective if and only if all its normal dual Banach left modules are 1-injective in the sense of the homology of Banach algebras. Nuclear $C^{*}$-algebras are characterized in a similar manner.
\end{abstract}

\section{INTRODUCTION}

Let $M$ be a unital Banach algebra. All Banach modules over $M$ will be assumed to be contractive and unital, and morphisms between them will be bounded linear maps commuting with the given actions. For any $a \in M$, let $L_{a}$ and $R_{a}$ be the corresponding left and right multiplication operators on $M$. With any Banach left $M$-module $X$ we associate a linear contraction $\alpha: X \rightarrow \mathcal{L}(M, X)$ by $(\alpha \xi)(x)=x \xi$, the action of $x \in M$ on $\xi \in X$, satisfying $\alpha(a \xi)=\alpha \xi \circ R_{a}$, for $\xi \in X$ and $a \in M$. $\mathcal{L}$ will denote the space of all bounded linear operators and $\widehat{\otimes}$ the projective tensor product of Banach spaces.

The following definition, due to M.C. White, [22, Definition 3.2, p.161], is a quantitative version of A.Ya. Helemskii's original definition; cf. [10, III. Definition 1.13, p.136] or [7, Definition 1.5, p.394].

Definition 1.1. Let $M$ be a Banach algebra, $X$ a Banach left $M$-module, and $c>0$ a constant. $X$ is called $c$-injective if the following holds: Given any morphism $\iota$ of Banach left $M$-modules admitting a bounded linear left inverse $l$, there is for any morphism $\varphi$ into $X$ a morphism $\tilde{\varphi}$ such that $\varphi=\tilde{\varphi} \circ \iota$ and $\|\tilde{\varphi}\| \leq c\|\varphi\|\|l\|$ :

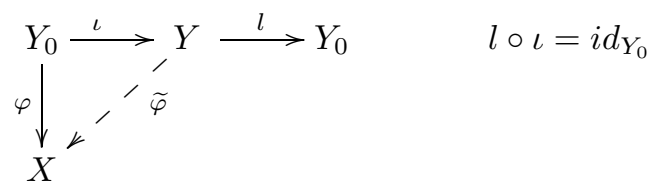

$X$ is called injective if there exists $\tilde{\varphi}$ satisfying just $\varphi=\tilde{\varphi} \circ \iota$. One obtains the definition of injective right and $A$-bimodules by replacing all morphisms and modules by their right and bi-versions, respectively.

The following criterion, also due to White, [22, Proposition 3.8, p.163], will be our main tool.

Received by the editors November 13, 2009 and, in revised form, July 9, 2010.

2010 Mathematics Subject Classification. Primary 46L10, 46L05, 46M10.

Key words and phrases. Injective von Neumann algebras, nuclear $C^{*}$-algebras, injective Banach modules.

(C)2010 American Mathematical Society 
Criterion 1.2. Let $M$ be a unital Banach algebra, $X$ a Banach left $M$-module with action $\alpha$, and $c>0 . X$ is c-injective if and only if there is a bounded linear mapping $\lambda$ of norm $\|\lambda\| \leq c$,

$$
X \stackrel{\alpha}{\longrightarrow} \mathcal{L}(M, X) \stackrel{\lambda}{\longrightarrow} X,
$$

satisfying $\lambda\left(T \circ R_{a}\right)=a(\lambda T)$ and $\lambda(\alpha \xi)=\xi$, for all $\xi \in X, T \in \mathcal{L}(M, X)$ and $a \in M$.

Example 1.3. Let $M$ be a Banach algebra with unit 1. The space, $B(M, M)$, of bounded bilinear forms on $M$ is a Banach $M$-bimodule under the norm

$$
\|V\|=\sup \{|V(x, y)|:\|x\| \leq 1,\|y\| \leq 1\},
$$

and the actions $(b V a)(x, y)=V(a x, y b)$, for $V \in B(M, M)$ and $a, b \in M$. Replacing $X$ by $B(M, M)$ in the diagram (1.1), we see that for any $\varphi$ the mapping $\tilde{\varphi}$,

$$
\tilde{\varphi}(\eta)(x, y)=\varphi(l(y \eta x))(1,1) \quad(\eta \in Y,(x, y) \in M \times M),
$$

satisfies $\varphi=\tilde{\varphi} \circ \iota$ and $\|\tilde{\varphi}\| \leq\|\varphi\|\|l\|$, so that the Banach $M$-bimodule $B(M, M)$ is 1-injective.

A von Neumann algebra $M$ acting on a Hilbert space $h$ is called injective if there is a bounded linear projection of norm 1 from $\mathcal{L}(h)$ onto $M$. In his papers 11 and [12], A.Ya. Helemskii discovered a deep relationship between the injectivity of a von Neumann algebra and the injectivity of some of its Banach modules. In particular, he proved in [12, Theorem, p.77], that all normal dual left Banach modules over an injective von Neumann algebra are injective in the sense of (1.1), leaving open the converse implication; cf. Problem 6 [13, p.164]. As a partial answer we have the following:

Corollary 2.6. A von Neumann algebra $M$ is injective if and only if all normal dual Banach left $M$-modules are 1-injective.

The constant 1 is significant in so far as it seems to be unknown at present whether the existence of any bounded linear projection from $\mathcal{L}(h)$ onto $M$ implies the injectivity of $M$; concerning this problem, see the papers by G. Pisier, [18, and E. Christensen and A.M. Sinclair, 4] and [5. Helemskii further showed, [11, Theorem 3, p.558], that $M$ is injective if and only if its predual, $M_{*}$, is an injective $M$-bimodule. The following is more precise.

Proposition 2.9. A von Neumann algebra $M$ is injective if and only if its predual Banach $M$-bimodule $M_{*}$ is 1-injective.

In the same vein we have the following characterizations of nuclear $C^{*}$-algebras, equally related to Helemskii's work; cf. [10, VII. Theorem 2.29, p.257] and [10, VII. Theorem 2.20, p.254], respectively.

Proposition 3.2. $A C^{*}$-algebra $A$ is nuclear if and only if all dual Banach left A-modules are 1-injective.

Proposition 3.4. $A C^{*}$-algebra $A$ is nuclear if and only if its dual Banach $A$ bimodule $A^{*}$ is 1-injective. 
Our proofs use Haagerup's characterization of injective von Neumann algebras, 9], and are, in fact, easy consequences of it. They are different from Helemskii's and constitute therefore also new proofs of his theorems quoted above. Although we formulate and prove (2.6) and (3.2) only for left modules, these are valid also for right modules. Let us finally recall the theorem of $\mathrm{A}$. Connes, according to which a von Neumann algebra $M$ is injective if and only if it is the $\sigma$-weak closure of the union of an increasing net of finite-dimensional $C^{*}$-subalgebras, each of which contains the identity of $M$, and that this property itself is equivalent to the amenability of $M$ in the sense of Johnson-Kadison-Ringrose, again by a theorem of Connes, [6]. For these and other equivalences we refer to Takesaki's third volume, 21.

\section{InJECTIVE VON NeUMANN ALGEBRAS}

2.1. Let us start with some notation. Let $M$ be a von Neumann algebra and $\pi: M \widehat{\otimes} M \rightarrow M$ be its multiplication. The predual, $M_{*}$, of $M$ is a norm closed subspace of the Banach space dual, $M^{*}$, of $M$ consisting of the $\sigma$-weakly continuous linear functionals on $M$; it is a Banach $M$-bimodule under the actions $(a f)(x)=$ $f(x a)$ and $(f a)(x)=f(a x)$, for $f \in M_{*}$ and $a, x \in M$. The bidual, $M^{* *}$, of $M$ can be made into a von Neumann algebra containing $M$ as a closed subalgebra which is $\sigma$-weakly, i.e. $\sigma\left(M^{* *}, M^{*}\right)$-dense in it. There is a unique projection, $z_{o}$, in the centre of $M^{* *}$ such that $M_{*}=M^{*} z_{o}$; cf. [20, III. Definition 2.13, p.127]. $B(M, M)=(M \widehat{\otimes} M)^{*}$ is the Banach space of all bounded bilinear forms on $M$, (1.3), and $B_{\sigma}(M, M)$ is the closed subspace consisting of the separately $\sigma$-weakly continuous ones.

A Banach left $M$-module $X$ will be called dual if there is a Banach right $M$ module $X_{*}$ whose dual left module, $\left(X_{*}\right)^{*}$, is isometrically isomorphic to $X$. In this case, $X$ is called normal if, for any $\xi \in X$ and $\varphi \in X_{*}$, the linear functional $\xi \varphi \in M^{*}$, defined by $\xi \varphi(x)=\xi(\varphi x)=(x \xi)(\varphi), x \in M$, actually belongs to $M_{*}$. If $X$ is any dual Banach left $M$-module, it becomes a left $M^{* *}$-module under the action, given by B.E. Johnson in [14, pp.16-17],

$$
\tilde{x} \xi(\varphi)=\tilde{x}(\xi \varphi), \quad \tilde{x} \in M^{* *}, \xi \in X \quad\left(\varphi \in X_{*}\right) .
$$

This action extends the action of $M$ on $X$ and, for fixed $\xi \in X$, the mapping $\tilde{x} \mapsto \tilde{x} \xi, \tilde{x} \in M^{* *}$, is $\sigma$-weak-weak* continuous from $M^{* *}$ into $X$.

Let $X$ be a dual Banach left $M$-module. For any bounded linear operator $T$ : $M \rightarrow X$, we denote by $\tilde{T}: M^{* *} \rightarrow X$ its unique $\sigma$-weak-weak* continuous extension, defined by $\tilde{T} \tilde{x}(\varphi)=\tilde{x}\left(T^{*} \varphi\right)$, for $\tilde{x} \in M^{* *}$ and $\varphi \in X_{*}$, having the same norm as $T$. The idea for the following lemma stems from [11, Lemma 2, p.559].

Lemma 2.2. Let $M$ be a von Neumann algebra and $X$ a normal dual Banach left $M$-module with predual $X_{*}$. Defining, for any $\varphi \in X_{*}$ and $T \in \mathcal{L}(M, X)$,

$$
\begin{aligned}
W_{\varphi, T}(\widetilde{x}, \widetilde{y}) & =\langle\varphi, \widetilde{x}(\widetilde{T} \widetilde{y})\rangle, & & (\widetilde{x}, \widetilde{y}) \in M^{* *} \times M^{* *}, \\
V_{\varphi, T}(x, y) & =W_{\varphi, T}\left(x z_{o}, y z_{o}\right), & & (x, y) \in M \times M,
\end{aligned}
$$

we have:

(i) The bounded bilinear forms $W_{\varphi, T}$ and $V_{\varphi, T}$ are separately $\sigma$-weakly continuous on $M^{* *}$ and $M$, respectively, and of norm $\leq\|\varphi\|\|T\|$ each;

(ii) $V_{\varphi, T \circ R_{a}}=\left(1 \widehat{\otimes} R_{a}\right)^{*} V_{\varphi, T}$ and $\left(L_{a} \widehat{\otimes} 1\right)^{*} V_{\varphi, T}=V_{\varphi a, T}$, for $a \in M$;

(iii) $V_{\varphi, \alpha \xi}=\pi^{*}(\xi \varphi)$, for $\xi \in X$. 
Proof of (i). Let us fix $\varphi \in X_{*}, T \in \mathcal{L}(M, X)$ and write $W_{\varphi, T}=W$ and $V_{\varphi, T}=$ $V$. Fix $\tilde{y} \in M^{* *}$ : for any net $\left(\tilde{x}_{\alpha}\right) \sigma$-weakly converging to $\tilde{x}$ in $M^{* *}$, the net $\left(\tilde{x}_{\alpha}(\tilde{T} \tilde{y})\right)$ converges weak ${ }^{*}$ to $\tilde{x}(\tilde{T} \tilde{y})$ s.t. $\lim W\left(\tilde{x}_{\alpha}, \tilde{y}\right)=W(\tilde{x}, \tilde{y})$. To prove the $\sigma$-weak continuity of $W$ in the second variable, we consider the bounded linear operator $w$,

$$
w: M^{* *} \rightarrow M^{* * *}, \quad(w \tilde{y})(\tilde{x})=W(\tilde{x}, \tilde{y}) \quad\left(\tilde{y}, \tilde{x} \in M^{* *}\right) .
$$

Since $W$ is $\sigma$-weakly continuous in the first variable, $w$ takes its values in $M^{*}$, and we will denote this operator by the same symbol $w: M^{* *} \rightarrow M^{*}, w(\tilde{y})=(\tilde{T} \tilde{y}) \varphi, \tilde{y} \in$ $M^{* *}$. $w$ being weakly compact by a theorem of Akemann, 1, Corollary II.9, p.293], the image of the unit ball of $M^{* *}, w\left(O M^{* *}\right)$, is relatively $\sigma\left(M^{*}, M^{* *}\right)$-compact, so that the restrictions of $\sigma\left(M^{*}, M^{* *}\right)$ and $\sigma\left(M^{*}, M\right)$ to $w\left(O M^{* *}\right)$ coincide. Fix $\tilde{x} \in M^{* *}$ : for any net $\left(\tilde{y}_{\alpha}\right)$ in $O M^{* *} \sigma$-weakly converging to $\tilde{y}$, the net $\left(\left(\tilde{T} \tilde{y}_{\alpha}\right) \varphi\right)$ converges to $(\tilde{T} \tilde{y}) \varphi$ in $\sigma\left(M^{*}, M\right)$. From $w(\tilde{y})=(\tilde{T} \tilde{y}) \varphi, \tilde{y} \in M^{* *}$, we conclude that $\left(w \tilde{y}_{\alpha}\right)$ converges to $w \tilde{y}$ in $\sigma\left(M^{*}, M^{* *}\right)$, so that $\lim W\left(\tilde{x}, \tilde{y}_{\alpha}\right)=\lim \left\langle w \tilde{y}_{\alpha}, \tilde{x}\right\rangle=$ $\langle w \tilde{y}, \tilde{x}\rangle=W(\tilde{x}, \tilde{y})$, entailing the $\sigma$-weak continuity of $W$ in the second variable.

The separate $\sigma$-weak continuity of $V$ is easy. Let us fix, for instance, $x \in M$. Since for any $f \in M^{*}$, we have $f z_{o} \in M_{*}$, the $\sigma$-weak convergence of $\left(y_{\alpha}\right)$ to $y$ in $M$ implies the $\sigma$-weak convergence of $\left(y_{\alpha} z_{o}\right)$ to $y z_{o}$ in $M^{* *}$ so that the continuity of $W$ in the second variable implies that $\lim V\left(x, y_{\alpha}\right)=V(x, y)$. Certainly, the norms of $W$ and $V$ do not exceed $\|\varphi\|\|T\|$.

Proof of (ii). Fix $\varphi \in X_{*}, T \in \mathcal{L}(M, X)$, and $a \in M$. Since $\left(T \circ R_{a}\right)^{\sim}(\tilde{y})=$ $\tilde{T}(\tilde{y} a), \tilde{y} \in M^{* *}$, we have for any $(x, y) \in M \times M$,

$$
\begin{aligned}
V_{\varphi, T \circ R_{a}}(x, y) & =W_{\varphi, T \circ R_{a}}\left(x z_{o}, y z_{o}\right)=\left\langle\varphi, x z_{o} \cdot \tilde{T}\left(y z_{o} a\right)\right\rangle \\
& =W_{\varphi, T}\left(x z_{o}, y a z_{o}\right)=V_{\varphi, T}(x, y a) \\
& =\left(1 \widehat{\otimes} R_{a}\right)^{*} V_{\varphi, T}(x, y)
\end{aligned}
$$

and

$$
\begin{aligned}
V_{\varphi a, T}(x, y) & =W_{\varphi a, T}\left(x z_{o}, y z_{o}\right)=\left\langle\varphi a, x z_{o} \cdot \tilde{T}\left(y z_{o}\right)\right\rangle \\
& =\left\langle\varphi, a x z_{o} \cdot \tilde{T}\left(y z_{o}\right)\right\rangle=W_{\varphi, T}\left(a x z_{o}, y z_{o}\right) \\
& =V_{\varphi, T}(a x, y)=\left(L_{a} \widehat{\otimes} 1\right)^{*} V_{\varphi, T}(x, y) .
\end{aligned}
$$

Proof of (iii). Let $\varphi \in X_{*}$ and $\xi \in X$. With $\alpha: X \rightarrow \mathcal{L}(M, X)$ defined by $\alpha \xi(x)=$ $x \xi$ for $x \in M$, we have $(\alpha \xi)^{*}(\varphi)=\xi \varphi$ for $\varphi \in X_{*}$, and hence $(\alpha \xi)^{\sim}(\tilde{x})=\tilde{x} \xi$, the action of $\tilde{x} \in M^{* *}$ on $\xi$. This gives, for any $(x, y) \in M \times M$,

$$
\begin{aligned}
V_{\varphi, \alpha \xi}(x, y) & =W_{\varphi, \alpha \xi}\left(x z_{o}, y z_{o}\right)=\left\langle\varphi, x z_{o} \cdot(\alpha \xi)^{\sim}\left(y z_{o}\right)\right\rangle \\
& =\left\langle\varphi, x z_{o} \cdot y z_{o} \xi\right\rangle=\left\langle\varphi, z_{o}(x y \xi)\right\rangle \\
& =\left\langle(x y \xi) \varphi, z_{o}\right\rangle=\left\langle x y(\xi \varphi), z_{o}\right\rangle \\
& =\left\langle(\xi \varphi) z_{o}, x y\right\rangle=\langle\xi \varphi, x y\rangle \\
& =(\xi \varphi) \circ \pi(x, y),
\end{aligned}
$$

since, by the normality of $X, \xi \varphi \in M_{*}$ and therefore $(\xi \varphi) z_{o}=\xi \varphi$. 
2.3. Let $\pi: M \widehat{\otimes} M \rightarrow M$ be the multiplication of the von Neumann algebra $M$. Its dual, $\pi^{*}: M^{*} \rightarrow(M \hat{\otimes} M)^{*}=B(M, M)$, maps $M_{*}$ into $B_{\sigma}(M, M)$, thus defining a map $\pi_{*}: M_{*} \rightarrow B_{\sigma}(M, M), \pi_{*} f(x, y)=f(x y)$, for $f \in M_{*}$ and $(x, y) \in M \times M$, the dual of which is $\left(\pi_{*}\right)^{*}: B_{\sigma}(M, M)^{*} \rightarrow M$. We have $\pi_{*}(b f a)=\left(L_{a} \widehat{\otimes} R_{b}\right)^{*}\left(\pi_{*} f\right)$, for $f \in M_{*}$ and $a, b \in M$, such that $\pi_{*}$ and $\left(\pi_{*}\right)^{*}$ are $M$-bimodule morphisms.

Proposition 2.4. Let $M$ be an injective von Neumann algebra. Then every normal dual Banach left $M$-module is 1-injective.

Proof. Let $X$ be a normal dual Banach left $M$-module, with predual $X_{*}$ and action $\alpha \xi(x)=x \xi$, for $\xi \in X$ and $x \in M$. By (1.2), it suffices to show that there exists a bounded linear map $\lambda: \mathcal{L}(M, X) \rightarrow X$ of norm one satisfying $\lambda\left(T \circ R_{a}\right)=a(\lambda T)$ and $\lambda(\alpha \xi)=\xi$, for all $\xi \in X, T \in \mathcal{L}(M, X)$ and $a \in M$. Let $I(M)$ be the set of isometries $u$ in $M, u^{*} u=1$. Since $M$ is injective, there exists by Haagerup's Theorem 2.1, 9, p.306], a state $m$ on $l^{\infty}(I(M))$ such that, for any separately $\sigma$ weakly continuous bilinear form $V$ on $M$ and all $a \in M$, we have

$$
\int V\left(a u^{*}, u\right) d m(u)=\int V\left(u^{*}, u a\right) d m(u) \quad\left(V \in B_{\sigma}(M, M), a \in M\right) .
$$

By defining

$$
\omega(V)=\int V\left(u^{*}, u\right) d m(u) \quad\left(V \in B_{\sigma}(M, M)\right),
$$

we obtain an element $\omega \in B_{\sigma}(M, M)^{*}$ of norm one and satisfying

$$
\left(L_{a} \widehat{\otimes} 1\right)^{* *} \omega=\left(1 \widehat{\otimes} R_{a}\right)^{* *} \omega, \quad\left(\pi_{*}\right)^{*} \omega=1 \quad(a \in M) ;
$$

cf. the proof of Theorem 3.1 in [9, p.312]. (Effros calls $\omega$ a normal virtual diagonal, [8. p.147], but Haagerup's $\omega$ has norm one.)

Now let $V_{\varphi, T} \in B_{\sigma}(M, M)$ be the bilinear form associated with $\varphi \in X_{*}$ and $T \in \mathcal{L}(M, X),(2.2)$. For $T$ fixed, $\varphi \mapsto \omega\left(V_{\varphi, T}\right)$ is a bounded linear functional on $X_{*}$ of norm $\leq\|T\|,(2.2(\mathrm{i}))$, such that there exists an element $\lambda T \in\left(X_{*}\right)^{*}=X$ of norm $\|\lambda T\| \leq\|T\|$ given by

$$
(\lambda T)(\varphi)=\omega\left(V_{\varphi, T}\right) \quad\left(T \in \mathcal{L}(M, X), \varphi \in X_{*}\right) .
$$

This defines a bounded linear map $\lambda: \mathcal{L}(M, X) \rightarrow X$ of norm $\leq 1$ and having the properties stated above. Indeed, we have, for any $a \in M$, by (2.2(ii)),

$$
\begin{aligned}
\lambda\left(T \circ R_{a}\right)(\varphi) & =\omega\left(V_{\varphi, T \circ R_{a}}\right)=\omega\left(\left(1 \hat{\otimes} R_{a}\right)^{*} V_{\varphi, T}\right) \\
& =\omega\left(\left(L_{a} \widehat{\otimes} 1\right)^{*} V_{\varphi, T}\right)=\omega\left(V_{\varphi a, T)}\right. \\
& =(\lambda T)(\varphi a)=(a(\lambda T))(\varphi)
\end{aligned}
$$

and, for any $\xi \in X$, by (2.2(iii)),

$$
\lambda(\alpha \xi)(\varphi)=\omega\left(V_{\varphi, \alpha \xi}\right)=\omega\left(\pi_{*}(\xi \varphi)\right)=\xi \varphi(1)=\xi(\varphi 1)=\xi(\varphi) \quad\left(\varphi \in X_{*}\right),
$$

since all modules were assumed to be unital.

2.5. The following lemma is proved in [19, 2.8. Lemma].

Lemma. Let $M$ be a Banach algebra, $X$ a Banach left $M$-module, and $c>0 a$ constant. If $X$ is c-injective, there exists a bounded linear projection, $P$, of norm $\|P\| \leq c$, from $\mathcal{L}(X)$ onto the subspace, $\mathcal{L}_{M}(X)$, of $M$-module morphisms of $X$.

Corollary 2.6. Let $M$ be a von Neumann algebra. $M$ is injective if and only if all normal dual Banach left $M$-modules are 1-injective. 
Proof. One direction has been proven in (2.4). The other one is proved in [19, 2.9. Corollary]. Let us repeat, for convenience, the brief argument. By letting $M$ act on a Hilbert space $h, h$ becomes a normal dual left $M$-module. If it is 1 -injective, then there is, by $(2.5)$, a linear projection of norm 1 from $\mathcal{L}(h)$ onto $\mathcal{L}_{M}(h)=M^{\prime}$, the commutant of $M$. Hence $M^{\prime}$ is injective, and so is $M$; cf. 21, XV. Proposition 3.2(iii), p.174].

2.7. Apart from the constant, the following proposition has been alluded to by Helemskii in [11, p.558], just before "Proof of Theorem 1".

Proposition. Let $M$ be a von Neumann algebra with predual $M_{*} . M$ is injective if and only if there is a bounded linear map $\lambda$,

$$
M_{*} \stackrel{\pi_{*}}{\longrightarrow} B_{\sigma}(M, M) \stackrel{\lambda}{\longrightarrow} M_{*},
$$

satisfying $\lambda\left(\left(L_{a} \hat{\otimes} R_{b}\right)^{*} V\right)=b(\lambda V)$ a and $\lambda\left(\pi_{*} f\right)=f$, for all $f \in M_{*}, V \in B_{\sigma}(M, M)$ and $a, b \in M$. If $M$ is injective, $\lambda$ can be chosen to be of norm one.

Proof. Let $M$ be injective and let $I(M)$ be the set of isometries in $M$. By Haagerup's Theorem 2.1, Lemmata 2.2 and 2.3, 9, pp.306-308], there exists a state $m$ on $l^{\infty}(I(M))$ such that, for any $V \in B_{\sigma}(M, M)$ and $a \in M$,

$$
\int V\left(a u^{*}, u\right) d m(u)=\int V\left(u^{*}, u a\right) d m(u) \quad\left(V \in B_{\sigma}(M, M), a \in M\right),
$$

and such that, for any $V \in B_{\sigma}(M, M)$, the linear functional $\lambda V$ on $M$,

$$
\lambda V(x)=\int V\left(x u^{*}, u\right) d m(u) \quad\left(V \in B_{\sigma}(M, M), x \in M\right),
$$

is $\sigma$-weakly continuous, i.e. $\lambda V \in M_{*}$. This defines a linear map $\lambda: B_{\sigma}(M, M) \rightarrow$ $M_{*}$ of norm $\leq 1$ and having the stated properties. Indeed, for any $V \in B_{\sigma}(M, M)$ and $a \in M$, we have

$$
\begin{aligned}
\lambda\left(\left(L_{a} \hat{\otimes} 1\right)^{*} V\right)(x) & =\int\left(L_{a} \hat{\otimes} 1\right)^{*} V\left(x u^{*}, u\right) d m(u) \\
& =\int V\left(a x u^{*}, u\right) d m(u) \\
& =(\lambda V)(a x) \\
& =((\lambda V) a)(x)
\end{aligned}
$$

and

$$
\begin{aligned}
\lambda\left(\left(1 \hat{\otimes} R_{a}\right)^{*} V\right)(x) & =\int\left(1 \hat{\otimes} R_{a}\right)^{*} V\left(x u^{*}, u\right) d m(u) \\
& =\int\left(1 \hat{\otimes} R_{a}\right)^{*} V\left(u^{*}, u x\right) d m(u) \\
& =\int V\left(u^{*}, u x a\right) d m(u) \\
& =\int V\left(x a u^{*}, u\right) d m(u) \\
& =(\lambda V)(x a) \\
& =(a(\lambda V))(x) \quad(x \in M)
\end{aligned}
$$

and, for any $f \in M_{*}$,

$$
\begin{aligned}
\lambda\left(\pi_{*} f\right)(x) & =\int \pi_{*} f\left(x u^{*}, u\right) d m(u) \\
& =\int f(x) d m(u) \quad(x \in M) . \\
& =f(x) \quad
\end{aligned}
$$


Conversely, let $\lambda: B_{\sigma}(M, M) \rightarrow M_{*}$ be any bounded linear map with the stated properties. Taking duals, we get a map $\lambda^{*}$,

$$
M \stackrel{\lambda^{*}}{\longrightarrow} B_{\sigma}(M, M)^{*} \stackrel{\left(\pi_{*}\right)^{*}}{\longrightarrow} M,
$$

satisfying $\lambda^{*}(a x b)=\left(L_{a} \widehat{\otimes} R_{b}\right)^{* *}\left(\lambda^{*} x\right)$ and $\left(\pi_{*}\right)^{*}\left(\lambda^{*} x\right)=x$, for any $x$ and $a, b$ in $M$, so that, with 1 the unit element of $M$,

$$
\omega=\lambda^{*}(1)
$$

is a normal virtual diagonal for $M$; cf. [8, p.147], since $\left(\pi_{*}\right)^{*} \omega=\left(\pi_{*}\right)^{*}\left(\lambda^{*} 1\right)=1$ and

$$
\left(L_{a} \widehat{\otimes} 1\right)^{* *} \omega=\left(L_{a} \widehat{\otimes} 1\right)^{* *} \lambda^{*}(1)=\lambda^{*}(a)=\left(1 \widehat{\otimes} R_{a}\right)^{* *} \lambda^{*}(1)=\left(1 \widehat{\otimes} R_{a}\right)^{* *} \omega \quad(a \in M) .
$$

By Effros's Theorem 3.1, [8, p.147], it follows that every $\sigma$-weak-weak* continuous derivation from $M$ into any normal dual Banach $M$-bimodule is inner and, by Connes's Theorem 1 [6, p.249], that $M$ is injective. (For another proof of the last implication, cf. [2, Corollary 4, p.235] or [4, Corollary 3.4, p.101].)

2.8. Let us recall that, for any von Neumann algebra $M, B(M, M)$ denotes the Banach space of all bounded bilinear forms on $M$ and $B_{\sigma}(M, M)$ denotes the closed subspace of separately $\sigma$-weakly continuous ones. Under the action $\left(L_{a} \widehat{\otimes} R_{b}\right)^{*} V(x, y)=V(a x, y b)$, for $V \in B(M, M)$ and $a, b \in M, B_{\sigma}(M, M)$ is a submodule of the Banach $M$-bimodule $B(M, M)$. The following is but a special case of [11, Lemma 2, p.559].

Lemma. Let $M$ be a von Neumann algebra. Then there exists a linear projection, $P_{\sigma}$, of norm one from $B(M, M)$ onto $B_{\sigma}(M, M)$ such that $P_{\sigma} \circ\left(L_{a} \widehat{\otimes} R_{b}\right)^{*}=$ $\left(L_{a} \widehat{\otimes} R_{b}\right)^{*} \circ P_{\sigma}$, for all $a, b \in M$.

Proof. Let $V \in B(M, M)$. It follows from [16, Theorem 2.3] that there exists a unique separately $\sigma$-weakly continuous bilinear form $\tilde{V}$ on $M^{* *}, \tilde{V} \in B_{\sigma}\left(M^{* *}, M^{* *}\right)$, extending $V$ and being of the same norm. With $z_{o} \in M^{* *}$ being the central projection satisfying $M_{*}=M^{*} z_{o}$, we define $P_{\sigma}$ on $B(M, M)$ by

$$
P_{\sigma} V(x, y)=\tilde{V}\left(x z_{o}, y z_{o}\right) \quad(V \in B(M, M),(x, y) \in M \times M) .
$$

Since $P_{\sigma} V$ is separately $\sigma$-weakly continuous, cf. the last paragraph of the proof of $(2.2(\mathrm{i}))$, we see that $P_{\sigma}$ is a linear contraction from $B(M, M)$ into $B_{\sigma}(M, M)$ commuting with all $\left(L_{a} \widehat{\otimes} R_{b}\right)^{*}$. It remains to show that $V=P_{\sigma} V$; i.e. $V(x, y)=$ $\tilde{V}\left(x z_{o}, y z_{o}\right), x, y \in M$, for all $V \in B_{\sigma}(M, M)$.

Let $V \in B_{\sigma}(M, M)$. Fix $x \in M$, and let $V_{x} \in M^{*}$ be defined by $V_{x}(y)=$ $V(x, y), y \in M$. For any $\tilde{y} \in M^{* *}$, we have, by choosing a net $\left(y_{\alpha}\right)$ in $M \sigma$-weakly converging to $\tilde{y}$ in $M^{* *}$,

$$
\left\langle V_{x}, \tilde{y}\right\rangle=\lim \left\langle y_{\alpha}, V_{x}\right\rangle=\lim \tilde{V}\left(x, y_{\alpha}\right)=\tilde{V}(x, \tilde{y}) \quad\left(x \in M, \tilde{y} \in M^{* *}\right),
$$

by virtue of the $\sigma$-weak continuity of $\tilde{V}$ in the second variable. $V_{x}$ belonging to $M_{*}$, by assumption, implies that $V_{x}=\left(V_{x}\right) z_{o}$, such that

$$
\tilde{V}(x, \tilde{y})=\left\langle V_{x}, \tilde{y}\right\rangle=\left\langle V_{x} z_{o}, \tilde{y}\right\rangle=\left\langle V_{x}, z_{o} \tilde{y}\right\rangle=\tilde{V}\left(x, \tilde{y} z_{o}\right) \quad\left(x \in M, \tilde{y} \in M^{* *}\right) .
$$

Now fix $\tilde{y} \in M^{* *}$, and let $\tilde{y} \tilde{V} \in M^{* * *}$ be defined by $\tilde{y} \tilde{V}(\tilde{x})=\tilde{V}(\tilde{x}, \tilde{y}), \tilde{x} \in M^{* *}$. It follows from the two-step construction of $\tilde{V}$ (and the $\sigma$-weak continuity of $V$ in 
the first variable) (cf. [16, Lemma 2.1, p.75]) that the restriction of $\tilde{y} \tilde{V}$ to $M$ is $\sigma$ weakly continuous, such that $\tilde{V}(x, \tilde{y})=\tilde{V}\left(x z_{o}, \tilde{y}\right)$, for all $x \in M$. For any $\tilde{x} \in M^{* *}$, we therefore obtain, for any net $\left(x_{\alpha}\right)$ in $M \sigma$-weakly converging to $\tilde{x}$ in $M^{* *}$,

$$
\tilde{V}(\tilde{x}, \tilde{y})=\lim \tilde{V}\left(x_{\alpha}, \tilde{y}\right)=\lim \tilde{V}\left(x_{\alpha} z_{o}, \tilde{y}\right)=\tilde{V}\left(\tilde{x} z_{o}, \tilde{y}\right) \quad\left(\tilde{x}, \tilde{y} \in M^{* *}\right)
$$

by virtue of the $\sigma$-weak continuity of $\tilde{V}$ in the first variable. With $\tilde{y} z_{o}$ instead of $\tilde{y}$, we get $\tilde{V}\left(\tilde{x}, \tilde{y} z_{o}\right)=\tilde{V}\left(\tilde{x} z_{o}, \tilde{y} z_{o}\right)$ and finally

$$
V(x, y)=\tilde{V}(x, y)=\tilde{V}\left(x, y z_{o}\right)=\tilde{V}\left(x z_{o}, y z_{o}\right) \quad(x, y \in M),
$$

proving that $V=P_{\sigma} V$, for any $V \in B_{\sigma}(M, M)$.

Proposition 2.9. Let $M$ be a von Neumann algebra with predual $M_{*}$. $M$ is injective if and only if the Banach $M$-bimodule $M_{*}$ is 1-injective.

Proof. Let the $M$-bimodule $M_{*}$ be just injective. Since the $M$-bimodule morphism $\pi_{*}: M_{*} \rightarrow B_{\sigma}(M, M)$ (cf. (2.3)) admits a bounded linear left inverse, $l$, given by $l V(x)=V(x, 1), x \in M$, there is, by (1.1), an $M$-bimodule morphism $\lambda$ completing the diagram

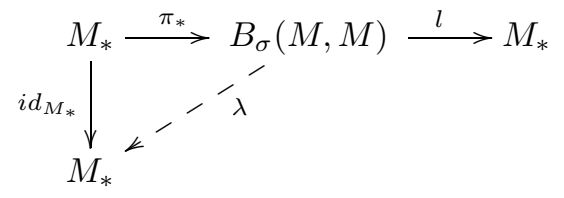

such that $\lambda \circ \pi_{*}=i d_{M_{*}}$, implying the injectivity of $M$, by (2.7). For the other direction we will copy Helemskii's argument, [11, p.565]. So let $M$ be injective. By (2.7), there are an $M$-bimodule morphism $\lambda$ of norm one such that $\lambda \circ \pi_{*}=i d_{M_{*}}$,

$$
M_{*} \stackrel{\pi_{*}}{\longrightarrow} B_{\sigma}(M, M) \stackrel{\lambda}{\longrightarrow} M_{*},
$$

and, by (2.8), an $M$-bimodule projection $P_{\sigma}$ of norm one from $B(M, M)$ onto $B_{\sigma}(M, M)$, such that $\left(\lambda \circ P_{\sigma}\right) \circ\left(\pi^{*} \mid M_{*}\right)=i d_{M_{*}}$,

$$
M_{*} \stackrel{\pi^{*} \mid M_{*}}{\longrightarrow} B(M, M) \stackrel{\lambda \circ P_{\sigma}}{\longrightarrow} M_{*} .
$$

$B(M, M)$ being 1-injective, by (1.3), the 1-injectivity of $M_{*}$ now follows easily using (1.1).

\section{NUCLEAR $C^{*}$-ALGEBRAS}

3.1. Let $A$ be a $C^{*}$-algebra and let, for any $a \in A, L_{a}$ and $R_{a}$ be the corresponding left and right multiplication operators on $A$. Identifying $(A \widehat{\otimes} A)^{*}$ with $B(A, A)$, the space of bounded bilinear forms on $A$, the dual of the multiplication of $A$, $\pi: A \widehat{\otimes} A \longrightarrow A$, is a mapping $\pi^{*}: A^{*} \longrightarrow B(A, A), \pi^{*} f(x, y)=f(x y), f \in A^{*}$, and satisfying $\pi^{*} \circ\left(L_{a}\right)^{*}=\left(L_{a} \widehat{\otimes} 1\right)^{*} \circ \pi^{*}, \pi^{*} \circ\left(R_{a}\right)^{*}=\left(1 \widehat{\otimes} R_{a}\right)^{*} \circ \pi^{*}, a \in A$, with $\left(L_{a} \widehat{\otimes} 1\right)^{*} V(x, y)=V(a x, y)$ and $\left(1 \widehat{\otimes} R_{a}\right)^{*} V(x, y)=V(x, y a)$, for $V \in B(A, A)$ and $a \in A$.

A Banach left $A$-module $X$ is called dual if there is a Banach right $A$-module $X_{*}$ whose dual left module is isometrically isomorphic to $X$. We shall assume that $X$ is faithful such that the action $\alpha: X \longrightarrow \mathcal{L}(A, X), \alpha \xi(x)=x \xi, \xi \in X, x \in A$, is injective; cf. [7, p.390]. This is equivalent to $X_{*}$ being essential, i.e. to the linear hull of the products $\varphi x, \varphi \in X_{*}, x \in A$, being dense in $X_{*}$.

Let us recall that the Banach space bidual, $A^{* *}$, of any $C^{*}$-algebra $A$ is a von Neumann algebra, the enveloping von Neumann algebra of $A$. By the theorem of 
Choi-Effros, 3], $A$ is nuclear if and only if $A^{* *}$ is injective, and this is the case if and only if $A$ is amenable, by the theorems of Connes and Haagerup, 6] and 9], already quoted.

Proposition 3.2. Let $A$ be a $C^{*}$-algebra. $A$ is nuclear if and only if every dual Banach left $A$-module is 1-injective.

Proof. Let $A$ be nuclear. By Haagerup's theorem, 9, Theorem 3.1, p.311], there exists a virtual diagonal of norm one for $A$, i.e. an element $\omega \in B(A, A)^{*}$ of norm $\|\omega\|=1$, satisfying $\omega \circ\left(L_{a} \widehat{\otimes} 1\right)^{*}=\omega \circ\left(1 \widehat{\otimes} R_{a}\right)^{*}$ and $\left(L_{a}\right)^{* *}\left(\pi^{* *} \omega\right)=\iota_{A}(a)$, for all $a \in A, \iota_{A}$ denoting the canonical embedding of $A$ into $A^{* *}$. We are going to define for any dual Banach left $A$-module $X$, with predual $X_{*}$, a bounded linear map $\lambda$ of norm $\|\lambda\|=1$,

$$
X \stackrel{\alpha}{\longrightarrow} \mathcal{L}(A, X) \stackrel{\lambda}{\longrightarrow} X,
$$

satisfying $\lambda\left(T \circ R_{a}\right)=a(\lambda T)$ and $\lambda(\alpha \xi)=\xi$ whenever $\xi \in X, T \in \mathcal{L}(A, X)$ and $a \in A$.

For any $\varphi \in X_{*}$ and $T \in \mathcal{L}(A, X)$, let $V_{\varphi, T}$ be defined by $V_{\varphi, T}(x, y)=\langle\varphi, x(T y)\rangle$, $(x, y) \in A \times A$, such that $V_{\varphi, T} \in B(A, A)$ is of norm $\left\|V_{\varphi, T}\right\| \leqslant\|\varphi\|\|T\|$. For $T$ fixed, the linear form $\varphi \longmapsto \omega\left(V_{\varphi, T}\right), \varphi \in X_{*}$, is bounded on $X_{*}$ by $\|T\|$ and determines therefore an element $\lambda T \in\left(X_{*}\right)^{*}=X$ by

$$
(\lambda T)(\varphi)=\omega\left(V_{\varphi, T}\right) \quad\left(T \in \mathcal{L}(A, X), \varphi \in X_{*}\right),
$$

of norm $\|\lambda T\| \leqslant\|T\|$. In this way, we obtain a linear contraction $\lambda$ from $\mathcal{L}(A, X)$ into $X$ with the properties stated. To see this, we observe, as in (2.2(ii)), that for any $a \in A$,

$$
\begin{aligned}
\lambda\left(T \circ R_{a}\right)(\varphi) & =\omega\left(V_{\varphi, T \circ R_{a}}\right) \\
& =\omega\left(\left(1 \widehat{\otimes} R_{a}\right)^{*} V_{\varphi, T}\right) \\
& =\omega\left(\left(L_{a} \widehat{\otimes} 1\right)^{*} V_{\varphi, T}\right) \\
& =\omega\left(V_{\varphi a, T}\right) \\
& =\lambda T(\varphi a) \\
& =(a(\lambda T))(\varphi),
\end{aligned}
$$

yielding the first one. To see the second, it suffices to assume $\varphi$ to be of the form $\varphi=\psi a$, for some $\psi \in X_{*}$ and $a \in A$, such that

$$
V_{\varphi, \alpha \xi}(x, y)=\langle\varphi, x . \alpha \xi(y)\rangle=\langle\varphi, x y \xi\rangle=\langle\psi, a(x y) \xi\rangle=\pi^{*}\left(L_{a}^{*} f\right)(x, y),
$$

with $f \in A^{*}$ defined by $f(x)=\langle\psi, x \xi\rangle, x \in A$, and hence

$$
\begin{aligned}
\lambda(\alpha \xi)(\varphi) & =\omega\left(V_{\varphi, \alpha \xi}\right) \\
& =\omega\left(\pi^{*} \circ\left(L_{a}\right)^{*} f\right) \\
& =\left(L_{a}\right)^{* *}\left(\pi^{* *} \omega\right)(f) \\
& =f(a) \\
& =\xi(\varphi),
\end{aligned}
$$

yielding the second property. The 1-injectivity of $X$ now follows from [7, Proposition 1.7, p.394].

For the converse, let $A$ act in its universal representation on the Hilbert space $h$ such that the $\sigma$-weak closure, $\bar{A}$, of $A$ in $\mathcal{L}(h)$ is isomorphic to $A^{* *}$. If the dual Banach left $A$-module $h$ is 1-injective, there exists by (2.5) a linear projection of 
norm one from $\mathcal{L}(h)$ onto $\mathcal{L}_{A}(h)=A^{\prime}$, the commutant of $A$, being equal to $(\bar{A})^{\prime}$, so that the commutant of $\bar{A}$ and hence of $A^{* *}$ is injective. This implies the injectivity of $A^{* *}$, 21, XV. Proposition 3.2 (iii), p.174], and therefore the nuclearity of $A$, by [3. Theorem 3, p.144] or [21, XV. Theorem 3.3, p.175].

Example 3.3. For any $C^{*}$-algebra $A$, the Banach $A$-bimodule, $B(A, A)$, of bounded bilinear forms on $A$ is 1-injective.

Indeed, let $\left(e_{\alpha}\right)$ be an approximate unit for $A$ of norm 1. Replacing in diagram (1.1) $X$ by $B(A, A)$, we define for any $\alpha$ a map $\varphi_{\alpha}: Y \longrightarrow B(A, A)$ by $\varphi_{\alpha}(\eta)(x, y)=$ $\varphi(l(y \eta x))\left(e_{\alpha}, e_{\alpha}\right), \eta \in Y$ and $x, y \in A$, such that $\left\|\varphi_{\alpha}\right\| \leqslant\|\varphi\|\|l\|\left\|e_{\alpha}\right\|^{2} \leqslant\|\varphi\|\|l\|$. Any weak *accumulation value, $\widetilde{\varphi}$, of $\left(\varphi_{\alpha}\right)$ in $\mathcal{L}(Y, B(A, A))=(Y \widehat{\otimes}(A \widehat{\otimes} A))^{*}$ is an $A$-bimodule morphism of norm $\|\widetilde{\varphi}\| \leqslant\|\varphi\|\|l\|$, satisfying $\varphi=\widetilde{\varphi} \circ \iota$. To prove the last equation, we take a subnet $\left(e_{\beta}\right)$ of $\left(e_{\alpha}\right)$ such that

$$
\widetilde{\varphi}(\eta)(x, y)=\lim \varphi(l(y \eta x))\left(e_{\beta}, e_{\beta}\right) \quad(\eta \in Y,(x, y) \in A \times A) .
$$

We then have, for any $\eta \in Y_{0}$,

$$
\begin{aligned}
\widetilde{\varphi}(\iota \eta)(x, y) & =\lim \varphi(l(y(\iota \eta) x))\left(e_{\beta}, e_{\beta}\right) \\
& =\lim \varphi(l \circ \iota(y \eta x))\left(e_{\beta}, e_{\beta}\right) \\
& =\lim \varphi(y \eta x)\left(e_{\beta}, e_{\beta}\right) \\
& =\lim (y \varphi(\eta) x)\left(e_{\beta}, e_{\beta}\right) \\
& =\lim \varphi(\eta)\left(x e_{\beta}, e_{\beta} y\right) \\
& =\varphi(\eta)(x, y) \quad((x, y) \in A \times A),
\end{aligned}
$$

i.e. $\widetilde{\varphi} \circ \iota=\varphi$. The bimodule property of $\widetilde{\varphi}$ follows directly from its definition.

Proposition 3.4. Let $A$ be a $C^{*}$-algebra. $A$ is nuclear if and only if its dual Banach A-bimodule $A^{*}$ is 1-injective.

Proof. Let us remark first of all that the $A$-bimodule morphism $\pi^{*}: A^{*} \longrightarrow$ $B(A, A)$ admits, for any $A$, a bounded linear left inverse $l$. With $\left(e_{\alpha}\right)$ an approximate unit for $A$ of norm 1, we define, for any $\alpha$, a linear contraction $l_{\alpha}$ : $B(A, A) \longrightarrow A^{*}$ by $l_{\alpha} V(x)=V\left(x, e_{\alpha}\right), V \in B(A, A)$ and $x \in A$. Then every weak *-accumulation value, $l$, of $\left(l_{\alpha}\right)$ in $\mathcal{L}\left(B(A, A), A^{*}\right)=(B(A, A) \widehat{\otimes} A)^{*}$ is a linear contraction from $B(A, A)$ into $A^{*}$ and satisfies, for some subnet $\left(e_{\beta}\right)$ of $\left(e_{\alpha}\right)$, $l\left(\pi^{*} f\right)(x)=\lim \pi^{*} f\left(x, e_{\beta}\right)=\lim f\left(x e_{\beta}\right)=f(x)$, for all $f \in A^{*}$ and $x \in A$, s.t. $l \circ \pi^{*}=i d_{A^{*}}$.

If $A^{*}$ is injective, there exists, by definition (1.1), an $A$-bimodule morphism $\lambda$,

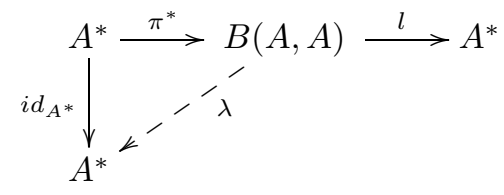

such that $\lambda \circ \pi^{*}=i d_{A^{*}}, \lambda \circ\left(L_{a} \widehat{\otimes} 1\right)^{*}=\left(L_{a}\right)^{*} \circ \lambda$, and $\lambda \circ\left(1 \widehat{\otimes} R_{a}\right)^{*}=\left(R_{a}\right)^{*} \circ \lambda$ whenever $a \in A$. Its dual map, $\lambda^{*}: A^{* *} \longrightarrow B(A, A)^{*}$, maps the unit element 1 of $A^{* *}$ onto a virtual diagonal $\omega=\lambda^{*}(1)$ for $A$, since, as in the proof in (2.7),

$$
\left(L_{a} \widehat{\otimes} 1\right)^{* *} \omega=\left(L_{a} \widehat{\otimes} 1\right)^{* *}\left(\lambda^{*} 1\right)=\lambda^{*}(a)=\left(1 \widehat{\otimes} R_{a}\right)^{* *}\left(\lambda^{*} 1\right)=\left(1 \widehat{\otimes} R_{a}\right)^{* *} \omega \quad(a \in A)
$$

and

$$
\pi^{* *} \omega=\pi^{* *}\left(\lambda^{*} 1\right)=1
$$


It follows from Johnson's Theorem 1.3, 15, p.688], that $A$ is amenable and therefore nuclear, by [6, Corollary 2, p.249].

Let, conversely, $A$ be nuclear. By [9, Theorem 3.1, p.311], there exists a virtual diagonal for $A$ of norm one, i.e. an element $\omega \in B(A, A)^{*}$ of norm $\|\omega\|=1$, satisfying $\omega \circ\left(L_{a} \widehat{\otimes} 1\right)^{*}=\omega \circ\left(1 \widehat{\otimes} R_{a}\right)^{*}$ and $\left(L_{a}\right)^{* *}\left(\pi^{* *} \omega\right)=\iota_{A}(a)$, for all $a \in A, \iota_{A}$ denoting the canonical embedding of $A$ into $A^{* *}$. Defining for any $V \in B(A, A)$, $\lambda V$ as in (2.7) by

$$
(\lambda V)(x)=\omega\left(\left(L_{x} \widehat{\otimes} 1\right)^{*} V\right) \quad(V \in B(A, A), x \in A),
$$

we obtain an element $\lambda V \in A^{*}$ of norm $\|\lambda V\| \leq\|V\|$, s.t. $\lambda$ defines a linear contraction,

$$
A^{*} \stackrel{\pi^{*}}{\longrightarrow} B(A, A) \stackrel{\lambda}{\longrightarrow} A^{*}
$$

satisfying $\lambda \circ\left(L_{a} \widehat{\otimes} 1\right)^{*}=\left(L_{a}\right)^{*} \circ \lambda, \lambda \circ\left(1 \widehat{\otimes} R_{a}\right)^{*}=\left(R_{a}\right)^{*} \circ \lambda$ whenever $a \in A$, and $\lambda \circ \pi^{*}=i d_{A^{*}}$. Indeed, we have for any $V \in B(A, A)$ and $a \in A$,

$$
\begin{array}{rlr}
\lambda\left(\left(L_{a} \widehat{\otimes} 1\right)^{*} V\right)(x) & =\omega\left(\left(L_{x} \widehat{\otimes} 1\right)^{*}\left(L_{a} \widehat{\otimes} 1\right)^{*} V\right) & \\
& =\omega\left(\left(L_{a x} \widehat{\otimes} 1\right)^{*} V\right) & \\
& =\lambda V(a x) & \\
& =((\lambda V) a)(x)
\end{array} \quad(x \in A),
$$

and

$$
\begin{array}{rlr}
\lambda\left(\left(1 \widehat{\otimes} R_{a}\right)^{*} V\right)(x) & =\omega\left(\left(L_{x} \widehat{\otimes} 1\right)^{*}\left(1 \widehat{\otimes} R_{a}\right)^{*} V\right) & \\
& =\omega\left(\left(1 \widehat{\otimes} R_{a}\right)^{*}\left(L_{x} \widehat{\otimes} 1\right)^{*} V\right) & \\
& =\omega\left(\left(L_{a} \widehat{\otimes} 1\right)^{*}\left(L_{x} \widehat{\otimes} 1\right)^{*} V\right) & \\
& =\omega\left(\left(L_{x a} \widehat{\otimes} 1\right)^{*} V\right) & \\
& =\lambda V(x a) & (x \in A),
\end{array}
$$

and finally, for $f \in A^{*}$,

$$
\begin{aligned}
\lambda\left(\pi^{*} f\right)(x) & =\omega\left(\left(L_{x} \widehat{\otimes} 1\right)^{*} \pi^{*} f\right) \\
& =\omega\left(\pi^{*}\left(L_{x}\right)^{*} f\right) \\
& =\left\langle f,\left(L_{x}\right)^{* *}\left(\pi^{* *} \omega\right)\right\rangle \\
& =f(x) \quad(x \in A) .
\end{aligned}
$$

$B(A, A)$ being 1-injective, by (3.3), the 1-injectivity of $A^{*}$ now follows directly from definition (1.1).

\section{Some REMARKS}

4.1. Let $M$ be an injective von Neumann algebra, $X$ a normal dual Banach left $M$-module and $\lambda: \mathcal{L}(M, X) \longrightarrow X$ the left inverse of the action, $\alpha$, of $M$ on $X$, as constructed in (2.3). For any $T \in \mathcal{L}(M, X), \lambda T$ is contained in the weak*-closed convex hull of the vectors $\left(u z_{0}\right)^{*} \widetilde{T}\left(u z_{0}\right), u \in I(M)$, the isometries in $M$, with $z_{0}$ being the central projection in $M^{* *}$ projecting $M^{*}$ onto $M_{*}$.

Indeed, if this were not the case, there would exist $T \in \mathcal{L}(M, X), \varphi \in X_{*}$ and a real number $c$ such that

$$
\operatorname{Re}\left\langle\varphi,\left(u z_{0}\right)^{*} \widetilde{T}\left(u z_{0}\right)\right\rangle \leqslant c<\operatorname{Re}\langle\varphi, \lambda T\rangle \quad(u \in I(M)),
$$


contrary to the definition of $\lambda T$,

$$
\langle\varphi, \lambda T\rangle=\int_{I(M)}\left\langle\varphi,\left(u z_{0}\right)^{*} \widetilde{T}\left(u z_{0}\right)\right\rangle d m(u) .
$$

4.2. Let $A$ be a nuclear $C^{*}$-algebra, $X$ a dual Banach left $A$-module and $\lambda$ : $\mathcal{L}(A, X) \longrightarrow X$ the left inverse of the action, $\alpha$, of $A$ on $X$, as constructed in (3.2). For any $T \in \mathcal{L}(A, X), \lambda T$ is contained in the weak*-closed convex hull of $\left\{a^{*}(T a): a \in A,\|a\| \leqslant 1\right\}$.

Let, for $T \in \mathcal{L}(A, X), \tau: A \widehat{\otimes} A \longrightarrow X$ be defined by $\tau(x \otimes y)=x(T y), x$ and $y$ in $A$, such that for its weak*-weak*-continuous extension $\widetilde{\tau}:(A \widehat{\otimes} A)^{* *} \longrightarrow X$ we have $\widetilde{\tau}(\omega)=\lambda T, \omega$ a virtual diagonal for $A$. Since, by [9, Theorem 3.1, p.311], $\omega$ can be chosen in $w^{*}-\operatorname{co}\left\{a^{*} \otimes a:\|a\| \leqslant 1\right\}$, we obtain $\lambda T \in w^{*}-\operatorname{co}\left\{\widetilde{\tau}\left(a^{*} \otimes a\right)=\right.$ $\left.a^{*}(T a):\|a\| \leqslant 1\right\}$.

4.3. Let $A$ be an amenable Banach algebra. If $A$ has a virtual diagonal of norm $d$, then every faithful dual Banach left $A$-module is $d$-injective. The qualitative part is a theorem of Helemskii, [10, VII. Theorem 2.29, p.257], and the quantitative part follows immediately from the first half of the proof of (3.2).

4.4. Let $A$ be a Banach algebra with a bounded two-sided approximate unit of norm c. Then $A$ is amenable if and only if its dual Banach $A$-bimodule $A^{*}$ is injective; cf. [10, VII. Theorem 2.20, p.254] and [10, VII. Theorem 1.14, p.243]. If $A$ has a virtual diagonal of norm $d$, then $A^{*}$ is $c^{2} d$-injective.

The proof of (3.3) shows that the $A$-bimodule $B(A, A)$ is $c^{2}$-injective. $\omega$ being a virtual diagonal for $A$ of norm $\|\omega\|=d$, we see, as in the proof of (3.4), that $A^{*}$ is $c^{2} d$-injective.

4.5. In contrast to (2.6), it does not seem possible to detect the injectivity of a von Neumann algebra within the category of its operator left modules and completely bounded (c.b.) morphisms. Indeed, let $A$ be any unital $C^{*}$-algebra acting on a Hilbert space $h$ and $h_{c}$ be the associated column operator space s.t. $h_{c}$ becomes an operator left $A$-module. Then for any completely isometric morphism $\iota$ between operator left $A$-modules $Y_{0}$ and $Y$ and any c.b. left $A$-morphism $\varphi$ there is a c.b. left $A$-morphism $\widetilde{\varphi}$ s.t. $\varphi=\widetilde{\varphi} \circ \iota$ and $\|\varphi\|_{c b}=\|\widetilde{\varphi}\|_{c b}$,

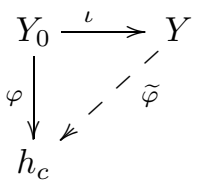

cf. Proposition 5.7 in [17, p.377]. This remark originates from a question of the referee, and I am very grateful to Professor Z-J. Ruan for providing me with the answer.

\section{ACKNOWLEDGEMENT}

The work for this paper was done at the Erwin Schrödinger International Institute for Mathematical Physics in Vienna during the workshop "Amenability beyond Groups". 


\section{REFERENCES}

[1] C.A. Akemann, The dual space of an operator algebra, Trans. Amer. Math. Soc. 126 (1967), 286-302. MR0206732 (34:6549)

[2] J.W. Bunce and W.L. Paschke, Quasi-expectations and amenable von Neumann algebras, Proc. Amer. Math. Soc. 71 (1978), 232-236. MR0482252 (58:2330)

[3] M.D. Choi and E.G. Effros, Nuclear $C^{*}$-algebras and injectivity: the general case, Indiana Univ. Math. Jour. 26 (1977), 443-446. MR0430794 (55:3799)

[4] E. Christensen and A.M. Sinclair, On von Neumann algebras which are complemented subspaces of $B(H)$, J. Funct. Anal. 122 (1994), 91-102. MR.1274585 (95f:46101)

[5] E. Christensen and A.M. Sinclair, Module mappings into von Neumann algebras and injectivity, Proc. London Math. Soc. (3) 71 (1995), 618-640. MR1347407 (96m:46107)

[6] A. Connes, On the cohomology of operator algebras, J. Funct. Anal. 28 (1978), 248-253. MR.0493383 (58:12407)

[7] H.G. Dales and M.E. Polyakov, Homological properties of modules over group algebras, Proc. London Math. Soc. (3) 89 (2004), 390-426. MR2078704 (2005e:46085)

[8] E.G. Effros, Amenability and virtual diagonals for von Neumann algebras, J. Funct. Anal. 78 (1988), 137-153. MR937636 (89e:46072)

[9] U. Haagerup, All nuclear $C^{*}$-algebras are amenable, Invent. Math. 74 (1983), 305-319. MR.723220 (85g:46074)

[10] A.Ya. Helemskii, The homology of Banach and topological algebras, Izdat. Moskow. Gos. Univ., Moscow (1986); English transl., Kluwer, Dordrecht (1989). MR.1093462 (92d:46178)

[11] A.Ya. Helemskii, The homological essence of Connes amenability: Injectivity of the predual bimodule, Matem. Sbornik 180 (1989), 1680-1690 (Russian); Math. USSR Sbornik 68 (1991), 555-566 (English). MR:1038222 (91f:46102)

[12] A.Ya. Helemskii, The spatial flatness and injectiveness of Connes operator algebras, Extracta Math. 9 (1994), 75-81. MR.1304157 (95i:46103)

[13] A.Ya. Helemskii, Some aspects of topological homology since 1995: a survey, Contemp. Math., 363, Amer. Math. Soc., Providence, RI, 2004, 145-179. MR2097957 (2005i:46079)

[14] B.E. Johnson, Cohomology in Banach algebras, Mem. Amer. Math. Soc. 127 (1972). MR 0374934 (51:11130)

[15] B.E. Johnson, Approximate diagonals and cohomology of certain annihilator Banach algebras, Amer. J. Math. 94 (1972), 685-698. MR0317050 (47:5598)

[16] B.E. Johnson, R.V. Kadison and J.R. Ringrose, Cohomology of operator algebras. III. Reduction to normal cohomology, Bull. Soc. Math. France 100 (1972), 73-96. MR0318908 $(47: 7454)$

[17] V.I. Paulsen, Relative Yoneda cohomology for operator spaces, J. Funct. Anal. 157 (1998), 358-393. MR.1638320 (99j:46067)

[18] G. Pisier, The operator Hilbert space OH, complex interpolation and tensor norms, Mem. Amer. Math. Soc. 122 (1996). MR.1342022 (97a:46024)

[19] G. Racher, On the projectivity and flatness of some group modules, Banach Center Publications 91 (2010).

[20] M. Takesaki, Theory of operator algebras. I, Springer-Verlag, New York, 1979. MR.548728 (81e:46038)

[21] M. Takesaki, Theory of operator algebras. III, Springer-Verlag, Berlin, 2003. MR 1943007 (2004g:46080)

[22] M.C. White, Injective modules for uniform algebras, Proc. London Math. Soc. (3) 73 (1996), 155-184. MR1387086 (97e:46068)

Universität Salzburg, Hellbrunnerstrasse 34, A-5020 Salzburg, Austria

E-mail address: gerhard.racher@sbg.ac .at 